Research Paper

\title{
Oncoplastic breast reconstruction with omental flap: A retrospective study and systematic review
}

\author{
Chao $\mathrm{Ni}^{1,2^{*}}$, Ziguan Zhu ${ }^{3 *}$, Yin Xin ${ }^{1}$, Qingping $\mathrm{Xie}^{3}$, Hongjun Yuan ${ }^{1}$, Miaochun Zhong1, Wenjie Xia1, \\ Xiaoyan $\mathrm{Zhu}^{4}$, Zhengye $\mathrm{Lv}^{1 凶}$, Xiangyang Song ${ }^{1 凶}$ \\ 1. Department of Breast and Thyroid Surgery, Zhejiang Provincial People's Hospital, People's Hospital of Hangzhou Medical College, Hangzhou, Zhejiang \\ 310014, P.R. China. \\ 2. Key Laboratory of Tumor Molecular Diagnosis and Individualized Medicine of Zhejiang Province. \\ 3. Department of Hand Surgery, Zhejiang Provincial People's Hospital, People's Hospital of Hangzhou Medical College, Hangzhou, Zhejiang 310014, P.R. \\ China. \\ 4. Department of General Surgery, Haining Central Hospital, Haining, Zhejiang 314408, P.R. China. \\ ${ }^{*}$ Co-first-authors
}

$\triangle$ Corresponding authors: Zhengye Lv, M.D., and Xiangyang Song, M.D., Ph.D.; Department of Breast and Thyroid Surgery, Zhejiang Provincial People's Hospital, People's Hospital of Hangzhou Medical College, Hangzhou, Zhejiang 310014, P.R. China. Tel.: $\pm 86-0571-85893383$; E-mail: lvzhenye@163.com, sxysong@163.com

(c) Ivyspring International Publisher. This is an open access article distributed under the terms of the Creative Commons Attribution (CC BY-NC) license (https://creativecommons.org/licenses/by-nc/4.0/). See http://ivyspring.com/terms for full terms and conditions.

Received: 2018.02.14; Accepted: 2018.03.05; Published: 2018.04.19

\begin{abstract}
The increasing frequency of early breast cancer cases has driven an increasing number of patients to choose immediate reconstruction with an autologous flap. Omentum-flap-based breast reconstruction is a unique strategy that is highly suitable for repairing moderate tissue defects. However, all available evidence comes from individual reports with small numbers of cases, and the overall effectiveness and safety of the procedure have yet to be reported. Here, we reported 7 cases of laparoscopically harvested omental free flap breast reconstruction and performed a systematic review to assess the applicability and safety of this approach. The data were gathered from MEDLINE, Ovid, Google Scholar and the China Knowledge Resource Integrated Database. In total, we combined 15 articles (410 cases) for analysis. The data revealed that almost all patients $(87.6 \%)$ were reported to have undergone laparoscopy instead of laparotomy; pedicle flaps were used in $90.9 \%$ of the cases, while only 5 (37 cases) used free flaps for reconstruction; and 96.6\% (396/410) of all reconstruction procedures were immediate. Almost all of these cases had a small tumour burden (T0/Tis/T1 59.8\%; T2 36.8\%), and the distribution of tumour location was similar among the four quadrants. The cosmetic outcomes were desirable in most cases (83.9\%). There were 41 complications identified in the dataset: partial graft necrosis accounted for the largest percentage $(41.5 \%)$ of all events, followed by skin necrosis (19.5\%), haematoma (12.2\%) and wound infection (9.8\%). During the follow-up period, which had a short median duration, 2 cases of tumour recurrence were reported. Overall, our systematic review found that omentum-flap-based breast reconstruction could achieve a satisfactory aesthetic outcome, especially for small breasts and tissue replacement after breast-conserving surgery, and the safety of the procedure was also acceptable.
\end{abstract}

Key words: Breast cancer; breast reconstruction; omentum

\section{Introduction}

The proportion of breast cancer cases detected in the early stages is increasing with the use of regular screening, and efforts to perform immediate breast reconstruction have become more common owing to the trend of less invasive surgery, such as breast-conserving surgery (BCS), nipple-sparing mastectomy and skin-sparing mastectomy[1]. Currently, immediate breast reconstruction can be achieved by use of various alloplastic or autologous materials. Autologous tissue reconstruction, in 
addition to being cost effective, can achieve a much more natural cosmetic result than the use of alloplastic materials because of the softness, warmth, and natural drop of the tissue; furthermore, autologous tissue is more durable against irradiation[2]. Currently, the most popular autologous flaps for breast reconstruction are latissimus dorsi (LD) and abdominal flaps, such as transverse rectus abdominis myocutaneous (TRAM) and deep inferior epigastric perforator (DIEP) flaps. However, there is still notable morbidity and deformity observed at the donor site, such as long scars, seroma and abdominal hernia risk.

The use of the omental flap is a unique technique that was first described in the 1880s for abdominal reconstruction[3]. The first report of breast reconstruction using the omental flap was in 1963[4]. This technique did not initially achieve popularity because of the severe laparotomy-associated complications that sometimes occurred. In 1998, however, Costa reported the performance of breast reconstruction with a laparoscopically harvested omental flap [5], and the advancement of laparoscopic techniques remarkably decreased the morbidity of the donor site. Although there have been increasing reports of this technique in the past two decades, almost all of them were single-centre case reports containing limited data and using pedicle omental flaps. In 2015, we shared our experience with 24 cases (pedicle omental flap)[6]. In the present study, we report another 7 cases of breast reconstruction with free omental flaps and perform the first systematic review of this technique in breast reconstruction.

\section{Materials and methods}

\section{Patients and indication}

Between 2016 and 2017, 7 patients underwent laparoscopically harvested omental free flap (LHOFF) breast reconstruction at Zhejiang Provincial People's Hospital, China. The indications for the 6 cases of BCS and 1 case of total breast reconstruction were delayed reconstruction with autologous tissue, considering that there was insufficient material for an abdominal flap.

\section{Surgical technique}

The process of omentum volume prediction and BCS was the same as previously reported[6]. The omentum was harvested laparoscopically by an experienced surgeon (Fig.S1A). The patients were placed in the supine position, and 3 trocars were inserted into the abdominal wall. The omentum was dissected in an avascular plane from the splenic flexure to the hepatic flexure of the colon. The left gastroepiploic artery and vein (GEAV) were carefully identified and dissected from the spleen. The right GEAV were also dissected cautiously and prepared for anastomosis. Next, the omentum was extracted through the trocar incision under the umbilicus and protected with a saline-soaked swab. Meanwhile, the other members of the surgical team prepared the ipsilateral thoracic dorsal artery and vein as the donor vessels. The gastroepiploic artery was anastomosed to the thoracic dorsal artery in an end-to-end fashion, and the accompanying vein was anastomosed using a flow coupler device (Synovis Micro Companies, USA, Fig. S1B). Next, the omental flap was clipped and shaped to the breast tissue defect for reconstruction.

\section{Postoperative evaluation}

The cosmetic evaluation system proposed by Kroll[7] was adopted. The cosmetic outcome was classified into four grades (excellent, good, fair, and poor) according to the four-category Harvard scale[8]. The surgeon and patients assessed the cosmetic outcome independently. Furthermore, the operating time, the length of hospitalization in days and any complications were all recorded. Moreover, irradiation was performed according to the National Comprehensive Cancer Network guidelines after surgery.

\section{Search strategy}

The literature search was performed by two reviewers independently with the MEDLINE, Ovid (including Embase 1974-2017) and Google Scholar databases and the China Knowledge Resource Integrated Database through November 2017. The key words applied were as follows: omentum, omental, reconstruction and breast. These search terms were adapted to the proper syntax for each database. The included manuscripts were restricted to the English and Chinese languages, and the titles and abstracts of publications identified by the search were examined manually to exclude reviews, letters and articles on topics not relevant to this study.

\section{Eligibility criteria}

Since we aimed to comprehensively summarize the published papers focusing on breast reconstruction for cosmetic purposes, cases involving the reconstruction of chest wall defects induced by trauma, surgical complications or irradiation were not included in this report. Both case reports and retrospective studies were accepted in this study. Articles with potential duplicate data were carefully analysed, and we included only the paper with the largest sample size. Articles without original data were also considered ineligible. 


\section{Data extraction}

All data were independently extracted by two reviewers according to a standardized method. For each study, the following information was extracted: author's name, publication year, number of patients, type of study, pathological result, tumour location (for BCS), tumour stage, axillary lymph node dissection (ALND), postoperative irradiation, breast surgery and reconstruction strategy, omentum harvest technique, omental flap type, cosmetic results, complications, patient complaints, hospital stay time, follow-up time and prognosis.

\section{Assessment of study quality}

The quality of all included publications was assessed by two independent reviewers according to the Grading of Recommendations Assessment, Development and Evaluation (GRADE) criteria. Studies with Newcastle-Ottawa Scale (NOS) scores above 6 were identified as high-quality studies, and disagreements were resolved by joint discussion.

\section{Statistical analysis}

The data for each measured variable were analysed. However, statistical meta-analysis could not be performed because of the low quality and great heterogeneity of the included studies.

\section{Results}

\section{Case report}

The patient characteristics are summarized in Table 1. All patients were diagnosed with breast cancer ( 6 cases of invasive ductal carcinoma and 1 case of ductal carcinoma in situ). Five patients underwent BCS with ALND and received immediate breast reconstruction with LHOFF; one patient underwent skin-sparing quadrantectomy and SLNB, followed by reconstruction with LHOFF. One patient had undergone mastectomy three years prior, and so we expanded the skin with an expander first and then replaced it with a free omental flap. The mean follow-up time was 9 months (3-13 months).

As shown in Table 1, the mean operation time was $311 \mathrm{~min}$ (270-370 $\mathrm{min})$, and the average time for omentum harvest was $81 \mathrm{~min}(65-100 \mathrm{~min})$. All excision margins were confirmed as negative. One patient presented with a wound infection one month after surgery. A bacterial culture revealed that the infectious agent was Staphylococcus lugdunensis, and part of the flap was lost with debridement. With the sole exception of this case, all cases were free of postoperative complications in the abdomen and breast. None of these patients felt pain, a dragging sensation or epigastric discomfort, and the wound at the donor site was minimal. Additionally, adjuvant irradiation was performed in all patients with BCS, and the shape of the breast was not affected. Neither local nor systemic recurrence has been found in any of the patients to date.

The aesthetic assessment by surgeons was excellent in 4 patients, good in 2 patients, and fair in 1 patient because of the loss of the partial flap. Aside from the patient who suffered from partial flap loss, the other 6 patients were all satisfied with the cosmetic results (Table S2).

\section{Search results}

In the initial search phase, we identified 108 publications (Figure 1). Thereafter, we manually checked all the publications by reading the abstract and full text, and we excluded 71 articles because they focused entirely on breast reconstruction. Eight articles were reviews or comments, 12 were missing the full text or original data, and 3 had duplicate datasets. The remaining 14 articles included 403 patients, and the 7 cases reported here were added for further systematic review (Table 2). Among the 14 published articles, there were 6 case reports[3, 9-13], 1 prospective study[14] and 7 retrospective series[6, 15-20]. Therefore, according to the GRADE system, all of the included studies were considered "low" or "very low" in quality.

Table 1. Patient characteristics

\begin{tabular}{|c|c|c|c|c|c|c|c|c|}
\hline Patient & Age (years) & Tumour type, stage and location & IR & Surgery & $\begin{array}{l}\text { Time for surgery/ } \\
\text { omentum harvest (min) }\end{array}$ & Complications & $\begin{array}{l}\text { Hospital stay } \\
\text { (days) }\end{array}$ & Follow-up (months) \\
\hline 1 & 34 & IDC, T2, LOU & Yes & BCS+ALND+LHOFF & $310 / 100$ & Uneventful & 7 & 13 \\
\hline 2 & 32 & IDC, T2, LOU & Yes & BCS+ALND+LHOFF & $270 / 65$ & Uneventful & 11 & 12 \\
\hline 3 & 43 & IDC, T3, LIL & Yes & BCS+ALND+LHOFF & $330 / 80$ & Uneventful & 20 & 10 \\
\hline 4 & 28 & IDC, T3, LOU & Yes & BCS+ALND+LHOFF & $370 / 90$ & Uneventful & 12 & 13 \\
\hline 5 & 53 & IDC, T2, LOU & Yes & BCS+ALND+LHOFF & $280 / 70$ & Graft infection & 14 & 6 \\
\hline 6 & 47 & DCIS, LOL & Yes & SSQ+SLNB+LHOFF & $320 / 85$ & Uneventful & 7 & 3 \\
\hline $7^{*}$ & 45 & IDC & No & 1) Expander, 2) LHOFF & $300 / 80$ & Uneventful & 7 & 7 \\
\hline
\end{tabular}

Abbreviations: IR, irradiation; IDC, invasive ductal carcinoma; DCIS, ductal carcinoma in situ; LOU, left outer upper; LIL, left inner lower; LOL, left outer lower; BCS, breast-conserving surgery; ALND, axillary lymph node dissection; SSQ, skin-sparing quadrantectomy; SLNB, sentinel lymph node biopsy; LHOFF, laparoscopically harvested omental free flap.

* The patient had undergone mastectomy several years earlier; we expanded the chest skin with an expander at the first stage and then replaced it with a free omental flap. 


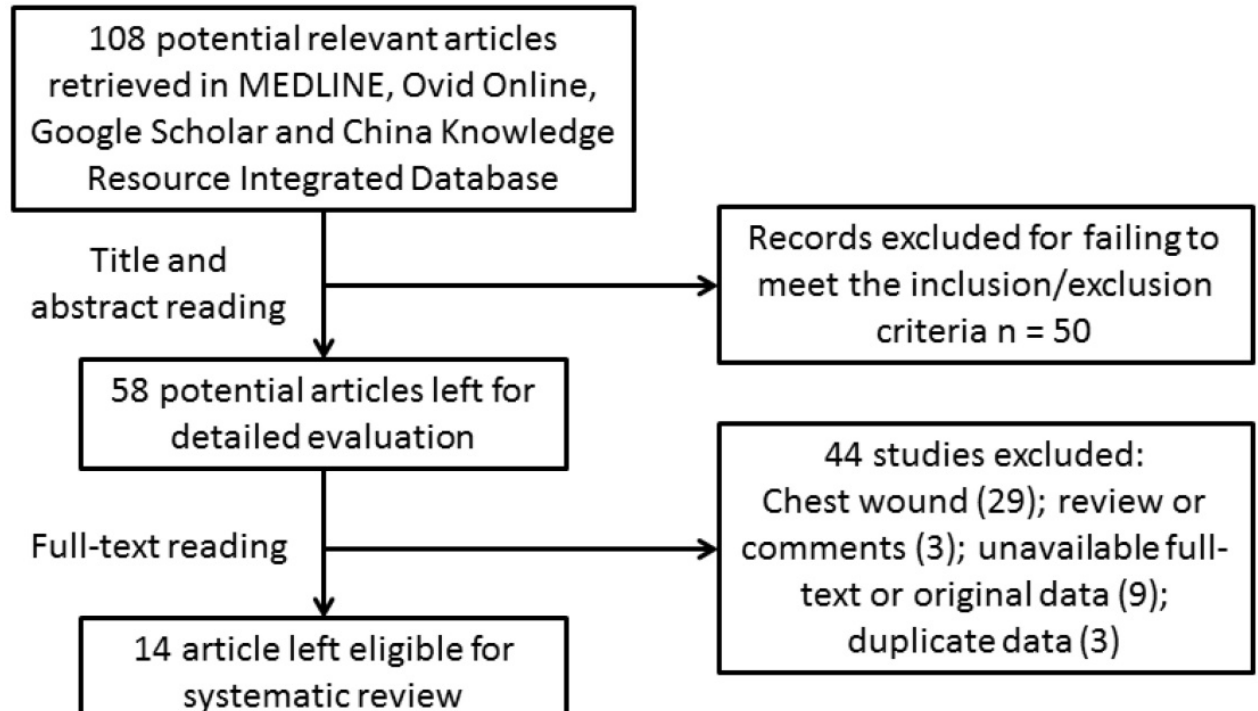

Figure 1. Flow chart of article selection.

Table 2. Characteristics of included studies

\begin{tabular}{|c|c|c|c|c|c|c|c|}
\hline Author & Year & Study design & Number & Flap design & Harvesting technique & Timing & Grade \\
\hline Arnold & 1977 & Case report & 2 & Pedicle & Laparotomy & Delayed & Very low \\
\hline McColl & 1979 & Case report & 2 & Pedicle & Laparotomy & Immediate & Very low \\
\hline Phillips & 1982 & Case report & 4 & Pedicle & Laparotomy & Delayed & Very low \\
\hline Baruch & 2001 & Retrospective study & 9 & Pedicle & Laparoscopy & Immediate & Low \\
\hline Lethbridge & 2002 & Case report & 4 & Free & Laparoscopy & $\begin{array}{l}\text { Immediate (3)/delayed } \\
\text { (1) }\end{array}$ & Very low \\
\hline Han & 2012 & Retrospective study & 19 & Pedicle & Laparotomy & Immediate & Low \\
\hline Khater & 2013 & Prospective study & 24 & Pedicle & Laparotomy & Immediate & Low \\
\hline Song & 2015 & Retrospective study & 24 & Pedicle & Laparoscopy & Immediate & Low \\
\hline Huang & 2015 & Retrospective study & 40 & Pedicle & Laparoscopy & Immediate & Low \\
\hline Kim & 2016 & Case series & 5 & Pedicle & Laparoscopy & Immediate & Very low \\
\hline Yu & 2016 & Retrospective study & 54 & Pedicle & Laparoscopy & Immediate & Low \\
\hline $\mathbf{L i}$ & 2017 & Retrospective study & 10 & Free & Laparoscopy & Immediate & Low \\
\hline Broekhuysen & 2017 & Case series & 6 & Pedicle & Laparoscopy & Delayed & Very low \\
\hline Zaha* & 2017 & Retrospective study & 200 & Free $(10) /$ pedicle $(190)$ & Laparoscopy & Immediate & Low \\
\hline Song & 2017 & Retrospective study & 7 & Free & Laparoscopy & Immediate & Low \\
\hline
\end{tabular}

* The same author published three articles in close succession; to avoid duplicated cases, we included only the publication with the largest number of cases.

\section{Technique evolution}

The first report in English of breast reconstruction with an omental flap was published in 1977[13], followed by 3 articles, including 8 cases, that were reported in the following five years[11-13]. Since laparoscopic surgery was developed and popularized in the late 1980s, all these cases used laparotomy for pedicle omentum harvest. No serious surgery-related complications were reported. In addition, in $75 \%$ of these cases (6/8), the omental flap was used as a covering for a suitable silicone gel prosthesis and as a support for an overlying thick split skin graft in delayed breast reconstruction[11, 13]. Subsequently, the application of omental flaps in tissue repair was primarily reported in chest wall defect reconstruction but not breast reconstruction until 2001[18]. In terms of publication time, Figure 2 reveals that the highest application rate of breast reconstruction with the large omentum occurred after the turn of the $21^{\text {st }}$ century.

\section{Surgical technique and clinical application}

The omentum harvest technique has undergone considerable refinement since 2001. Nearly all articles (10/12), including 359 patients, reported using laparoscopy instead of laparotomy. Moreover, a pedicle omental flap was the first choice for most surgeons, being used in $90.9 \%$ of all included cases (373/410), while only 5 articles $(37 / 410)$ reported using a free omental flap for reconstruction. Furthermore, $96.6 \%$ of these omental-flap-based surgeries were immediate (396/410), while only 14 cases were delayed surgeries after mastectomy, of which $57.1 \%(8 / 14)$ were reconstructed with the omentum plus an implant or expander. We also found that $59.1 \%$ of all immediate reconstruction $(234 / 396)$ cases occurred in BCS surgeries for partial tissue defect replacement, while $40.9 \%$ cases $(162 / 396)$ were for total breast reconstruction and $12.3 \%(20 / 162)$ were accompanied by an implant or LD flap (Table 2). 


\section{A}

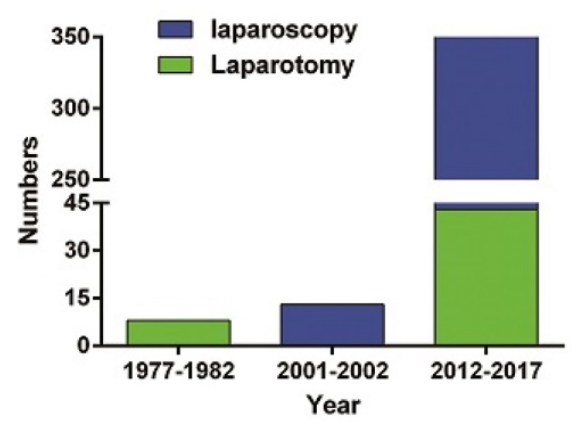

C

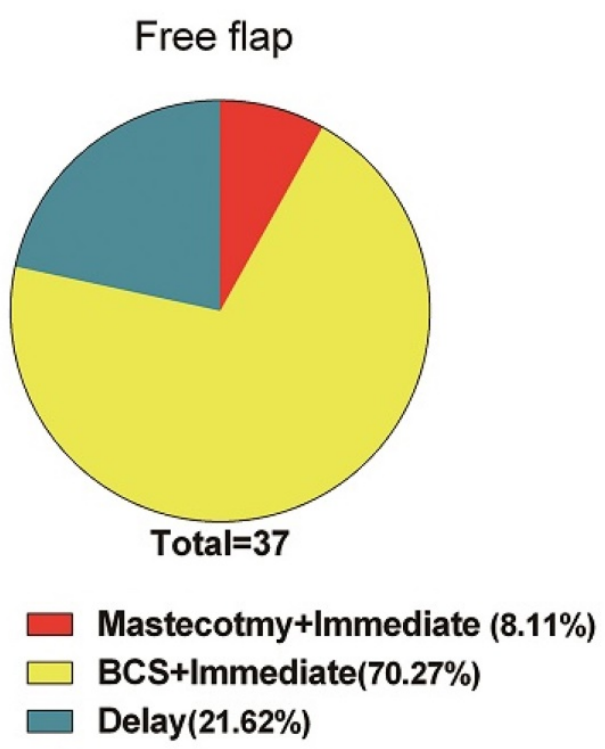

B

\section{Complications}

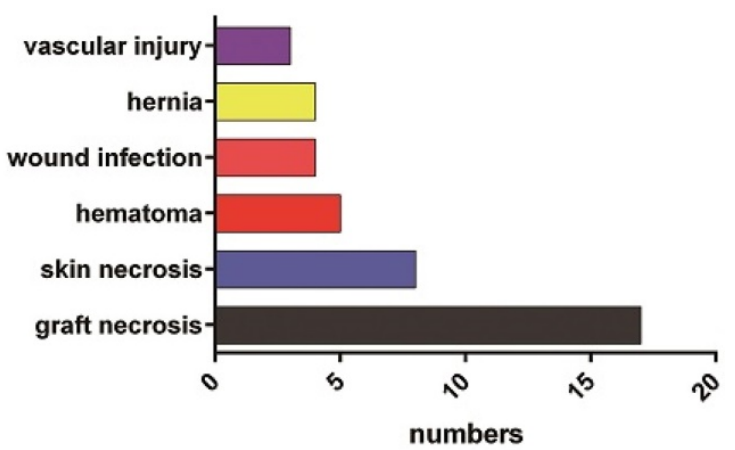

Pedicle flap

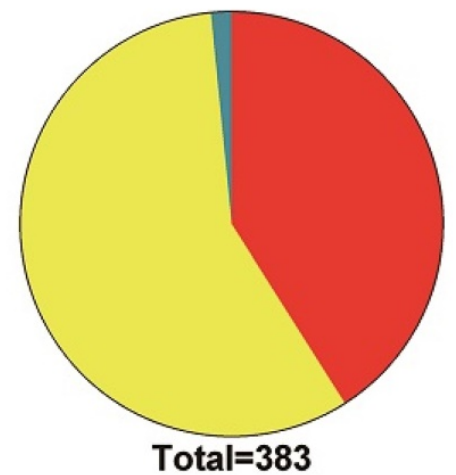

$\square$ Mastecotmy+Immediate (40.99\%)
$\square$ BCS+Immediate (57.44\%)
$\square$ Delay (1.57\%)

Figure 2. Publication years, complications and reconstructive strategies. A, publication years and omentum harvest techniques of the included articles; B, distribution of complications; C, distribution of strategies for omental-flap-based breast reconstruction (total, mastectomy, BCS)

Since the tumour stage, location and postoperative treatment are also highly important determinants of the breast reconstruction strategy, these factors were also taken into account in this study (Table 3). According to the available data, $19.6 \%$ of the cases were ductal carcinoma in situ (DCIS) (72/367), $77.9 \%$ were invasive breast carcinoma (286/367), and the rest were benign or precancerous lesions or congenital pathologies. Additionally, it seemed that the frequencies of different tumour locations were similar in patients undergoing omental flap reconstruction (outer upper quadrant, 36.3\% (98/270); outer lower quadrant, $16.3 \%$ (44/270); inner upper quadrant, $22.2 \%$ (60/270); and inner lower quadrant, $23.0 \%(62 / 270))$. Tumour size is also a key point in BCS. Almost all of the examined cases had small tumour burdens (T0/Tis/T1 59.8\% (156/261); T2 $36.8 \%(96 / 261))$, and only 9 cases $(9 / 261)$ had a tumour burden of T3 or higher. After surgery, most patients received radiotherapy $(80 \%, 204 / 254)$ if necessary. Relevant complications were rarely reported, with marked, permanent size reduction being reported in only two cases[15].

\section{Cosmetic outcomes}

Two early articles did not report cosmetic results[11, 13], while almost all of the remaining articles achieved desirable cosmetic outcomes. Specifically, $83.9 \%$ of the identified cases $(339 / 404)$ were evaluated as satisfactory, excellent or good, which indicates satisfactory bilateral symmetry (Table 4). The main reasons for unsatisfactory cosmetic results included intraoperative flap necrosis, partial flap loss during convalescence and volume insufficiency $[6,15,16]$. Moreover, several articles reported that the omentum could be very firm after surgery[3], which was also observed in this study, and there were temporary slight deformities after postoperative irradiation. Ultimately, however, those issues resolved on their own within several months without any intervention[6]. 
Table 3. Flap design and harvesting technique according to the oncological characteristics

\begin{tabular}{|c|c|c|c|c|c|c|c|c|}
\hline & Author & Tumour stage & Location* & $\operatorname{IR}(\%)^{* *}$ & Immediate (number) & Delayed & $\begin{array}{l}\text { Follow-up } \\
\text { (months) }\end{array}$ & Prognosis \\
\hline 1 & Arnold & NR & & NR & & $2^{a}$ & NR & NR \\
\hline 2 & McColl & NR & & NR & NSM (1); SSM (1) & & NR & NR \\
\hline 3 & Phillips & NR & & NR & & $4^{\text {a }}$ & NR & NR \\
\hline 4 & Baruch & NR & & NR & $\operatorname{SSM}\left(7+2^{a}\right)$ & & NR & NR \\
\hline 5 & Lethbridge & NR & & NR & SSM (3) & $1 \mathrm{~b}$ & NR & NR \\
\hline 6 & Han & NR & & $2(100)$ & NSM (15); SSM (4) & & $20(3-60)$ & Uneventful \\
\hline 7 & Khater & NR & & $6(100)$ & NSM (17); SSM (7) & & NR & NR \\
\hline 8 & Song & NR & $\begin{array}{l}\text { OU (14); OL (3); } \\
\text { IU (3); IL (5) }\end{array}$ & $24(100)$ & BCS (24) & & $32(6-51)$ & LR (1) \\
\hline 9 & Huang & $\begin{array}{l}\text { Tis/1 (26); } \\
\text { T2 (14) }\end{array}$ & $\begin{array}{l}\text { OU (14); OL (11); } \\
\text { IU (8); IL (7) }\end{array}$ & $40(100)$ & BCS (40) & & $15.6(6-36)$ & Uneventful \\
\hline 10 & Kim & $\begin{array}{l}\text { Tis } / 0 / 1(4) \text {; } \\
\text { T2 (1) }\end{array}$ & & $1(20)$ & SSM (3); NSM (2) & & $8.2(5-11)$ & Uneventful \\
\hline 11 & $\mathrm{Yu}$ & NR & & NR & NSM (54) & & $26.6(12-48)$ & Uneventful \\
\hline 12 & $\mathrm{Li}$ & T0/1 (1); T2 (9) & NR & $9(90)$ & BCS (10) & & NR & Uneventful \\
\hline 13 & Broekhuysen & NR & & NR & & $6^{a}$ & $30.5(3-80)$ & NR \\
\hline 14 & Zaha & $\begin{array}{l}\text { Tis/1 (125); } \\
\text { T2 (68)/T3 (7) }\end{array}$ & $\begin{array}{l}\text { OU (66); OL (29); } \\
\text { IU (49); IL (50); C (6) }\end{array}$ & $114(74)$ & $\begin{array}{l}\text { BCS (154); } \\
\text { NSM }\left(31+15^{a}\right)\end{array}$ & & $90(5-174)$ & LR (2) \\
\hline 15 & Song & T2 (4)/T3 (2) & OU (4); OL (1); IL (1) & $6(100)$ & BCS (6) & 1 & $9.1(3-13)$ & Uneventful \\
\hline
\end{tabular}

Table 4. Cosmetic results and clinical complications

\begin{tabular}{|c|c|c|c|c|c|c|}
\hline & Author & Numbers & Cosmetic results* & $\begin{array}{l}\text { Chief complaint } \\
\text { (numbers) }\end{array}$ & Complication & $\begin{array}{l}\text { Hospital stay } \\
\text { (days) }\end{array}$ \\
\hline 1 & Arnold & 2 & NR & Uneventful & Uneventful & NR \\
\hline 2 & McColl & 2 & Satisfactory & Uneventful & Uneventful & NR \\
\hline 3 & Phillips & 4 & NR & NR & Uneventful & NR \\
\hline 4 & Baruch & 9 & Satisfactory (7); fair (2) & $\begin{array}{l}\text { Epigastric } \\
\text { discomfort (1) }\end{array}$ & Partial skin necrosis (1) & NR \\
\hline 5 & Lethbridge & 4 & Satisfactory & NR & Uneventful & NR \\
\hline 6 & Han & 19 & $\begin{array}{l}\text { Excellent/good (16); } \\
\text { fair (3) }\end{array}$ & NR & Partial graft necrosis (1) & NR \\
\hline 7 & Khater & 24 & $\begin{array}{l}\text { Satisfactory/excellent (22); } \\
\text { fair (2) }\end{array}$ & NR & Vascular injury (1); wound infection (2); epigastric hernia (1); haematoma (2) & NR \\
\hline 8 & Song & 24 & Excellent (23); fair (1) & $\begin{array}{l}\text { Epigastric } \\
\text { discomfort (4) }\end{array}$ & Partial graft necrosis (1) & $9(7-14)$ \\
\hline 9 & Huang & 40 & $\begin{array}{l}\text { Excellent/good (39); } \\
\text { fair (1) }\end{array}$ & Uneventful & Partial graft necrosis (2) & $9.5(6-18)$ \\
\hline 10 & Kim & 5 & Excellent/good (5) & Uneventful & Uneventful & $11.2(8-14)$ \\
\hline 11 & $\mathrm{Yu}$ & 54 & $\begin{array}{l}\text { Excellent/good (48); } \\
\text { fair/poor (6) }\end{array}$ & NR & Partial skin necrosis (2) & NR \\
\hline 12 & $\mathrm{Li}$ & 10 & $\begin{array}{l}\text { Excellent/good (9); } \\
\text { fair (1) }\end{array}$ & Uneventful & Free flap necrosis (1); haematoma (1) & $7.7(4-20)$ \\
\hline 13 & Broekhuysen & 6 & Satisfactory (6) & $\begin{array}{l}\text { Occasional } \\
\text { stinging (1) }\end{array}$ & Flap necrosis (1); venous thrombosis (1) & NR \\
\hline 14 & Zaha & 200 & $\begin{array}{l}\text { Excellent/good (152); } \\
\text { fair/poor ( } 38)\end{array}$ & NR & $\begin{array}{l}\text { Vascular injury (2); wound infection (2); epigastric hernia (2); haematoma (2); } \\
\text { partial graft necrosis (10); partial skin necrosis (5) }\end{array}$ & NR \\
\hline 15 & Song & 7 & $\begin{array}{l}\text { Excellent/good (6); } \\
\text { poor (1) }\end{array}$ & NR & Graft infection (1) & $11.1(7-20)$ \\
\hline
\end{tabular}

Abbreviation: NR, not reported. * Interpretation was based on the original articles.

\section{Complications and oncological safety}

There were 41 complications identified in the 410 patients included (Table 4). Partial graft necrosis accounted for $40 \%$ of all events $(41.5 \%, 17 / 41)$, followed by skin necrosis $(19.5 \%, 8 / 41)$, haematoma $(12.2 \%, 5 / 41)$ and wound infection $(9.8 \%, 4 / 41)$. Abdominal intervention-related complications occurred in 7 cases $(17.1 \%)$, of which 3 were vascular injuries and 4 were abdominal hernias; notably, only two cases suffered from hernias with the laparoscopic technique $(0.6 \%, 2 / 359)$, which was considerably lower than the herniation rate for laparotomy ( $4 \%$, $2 / 51$ ). The scarcity of patient complaints indicated good tolerance, with only one case demonstrating slight occasional stinging and four cases reporting epigastric discomfort. The duration of hospitalization varied greatly among studies (7.7-11.2 days), which may be due to some surgeons discharging patients only after completion of all treatments, including suture removal and drainage removal[9].

Regarding oncological outcomes, there were 6 works with long follow-up results[3, 6, 15, 17, 19, 20]; 
those studies reported only 3 cases of tumour recurrence in total (Table 3), and all three cases occurred in the ipsilateral breast. Two of those cases were spared from radiotherapy after BCS, and both maintained disease-free survival after salvage surgery[15]. The other case was detected 6 months after surgery, and the patient passed away due to distant metastasis 5 months later[6].

\section{Discussion}

The literature examining the use and outcomes of the omentum in breast reconstruction is poorly described and outdated, and the present study provides the most current and comprehensive review on the reconstruction of mastectomy defects using the omentum.

Although the abdominal and LD flaps remain the most widespread and conventional selections for oncoplastic surgeons, as they provide considerably large tissue volume, an appropriate breast reconstructive method for slim patients with small breasts still needs to be determined, especially in Western countries[21]. In our experience, most of these patients lack sufficient autologous abdominal tissue and are reluctant towards the idea of large scars at the donor site. Additionally, implant-based reconstruction is usually not the first choice for small and ptotic breasts. In such cases, the omental flap could be an optimal choice for autologous tissue reconstruction when skin is preserved.

The main advantages of the omental flap include great malleability, vascular reliability, high absorptive ability against hydrops, potential local immune function and, most importantly, minimal donor-site morbidity, including low risk of conspicuous scars, haematoma, seroma and dragging sensations $[6,9,14$, $22,23]$. In the present review, nearly $10 \%$ of cases were reported to have complications, but most of the complications were insignificant and did not require medical intervention. The most frequent local complication was partial flap necrosis $(41.5 \%, 17 / 41)$, which usually appeared as hard nodules or indurations a few weeks after surgery and may have been due to fat necrosis[3, 6, 15]. In our experience, except for one case with serious infection that needed debridement, the patients all improved gradually with no cosmetic effects.

It is worth mentioning that in BCS, our data indicated that there were no selective differences in tumour location, and the cosmetic results were all acceptable. Medial-quadrant tissue defects, especially in the inner upper quadrant, which is known as 'no-man's land', are usually hard to reach with autologous tissue[24] but can be easily shaped by the omental flap as either a pedicle flap or a free flap. In our experience, a free omental flap, as opposed to a pedicle flap, can reach any part of the breast easily. For example, a free omental flap can reach the medial quadrant through a tunnel in the retro-mammary space with several stitches for fixation on the surrounding gland; furthermore, a free omental flap can repair axillary tissue defects very well after ALND (Figure S2). Our systematic review showed that the thoracodorsal vessel, rather than the inner mammary vessel, was usually the first choice of donor vessel[3, $10,15,16]$, since it is more familiar to the breast surgeon and easier to expose. Compared with the deep internal thoracic artery and thoracoacromial artery, the thoracic dorsal artery has a secluded location, stable anatomy, and similar diameter to the right artery of the stomach omentum, making it the usual first choice for us to perform anastomosis.

Although the omentum is easy to harvest, as it is a skinless flap, it was usually used in immediate breast reconstruction with skin-sparing surgery. Several surgeons thought the omentum was more suitable for BCS than for total breast reconstruction because of its undetermined volume. Neither body mass index nor radiological methods could provide precise information before surgery[6, 18, 25]. Zaha found that insufficiency could occur in more than $30 \%$ of the cases[15]. According to our systematic review, more than $40 \%(162 / 396)$ used the omentum for total breast reconstruction. This contradiction may be attributed to patient selection. In the largest case series reported to date, the author indicated that volume insufficiency could occur in partial reconstruction after BCS when the resection volume exceeds $200 \mathrm{~g}$, and in certain very thin patients, the omentum could be less than $100 \mathrm{~g}[15]$. According to the available data, the omentum weight of patients selected for breast reconstruction was almost 200-300 $\mathrm{g}[3,9,10,15]$, and in our experience, when perform total breast reconstruction with omentum, the breast volume should not exceeds $300 \mathrm{~g}$, which indicates patients with small breasts; and for partial tissue replacement, one should be careful if the estimated preoperative weight of the specimen is more than 300 g. with the above principle, in our reported 24 cases of pedicle omental breast reconstruction[6] and 7 cases free omental breast reconstruction, cosmetic results were almost satisfied and volume insufficiency have not happened.

Laparoscopy is considered an optimal method to evaluate the volume of the omentum, taking only 10-15 $\mathrm{min}$ and causing minimal wounding[3]. Moreover, we think it is important to have a thorough discussion with patients preoperatively regarding the inevitable use of implants or other autologous tissue, such as an LD flap, in cases of discrepant volume 
during operation. Furthermore, the data presented here reveal that only $3.4 \%$ of cases had delayed surgeries after mastectomy. Van Alphen et al.[3] reported three cases of LHOFF following mastectomy and used a subpectoral expander first to augment the skin, after which the expander was replaced with the omentum for reconstruction. In this study, we reported a similar case; this two-step strategy could be an ideal option for patients with breast ptosis and a small contralateral breast.

Concerning the oncological issue, several researchers have argued that adipocytes can stimulate the growth of breast cancer cells by increasing the local concentration of oestrogen, while some adipocytokines and the greater omentum may offer additional risks due to a high concentration of stem cells with great angiogenic potential[4, 26, 27]. However, this theory is not supported by the available clinical evidence. None of the individual researchers found an increased risk of cancer recurrence when the omentum was used for reconstruction. Here, there were only 2 cases of recurrence $(0.48 \%)$ during median follow-up periods of 32 months[6] and 78 months[15]. In addition, the radiolucent nature of the omentum, compared with the surrounding breast parenchyma, makes it conducive to follow-up after surgery.

\section{Conclusions}

Compared with implant usage or other autologous flaps, the omental flap has unique advantages for breast reconstruction in certain circumstances. This flap can achieve a better aesthetic outcome than other sources of material, especially for total reconstruction of small breasts and tissue replacement after BCS. Furthermore, laparoscopic techniques offer low morbidity at the donor site. However, there are still several limitations to our findings, as most of the studies were non-randomized and only one work had a follow-up time of more than 5 years[15]; consequently, further evidence is needed to establish the oncological safety of the procedure. Moreover, the problem of imprecise preoperational evaluation of the omental volume remains unsolved. Further studies are needed to assess the long-term safety of omental-flap-based breast reconstruction and to establish standard criteria for the procedure.

\section{Abbreviations}

ALND, axillary lymph node dissection; BCS, breast-conserving surgery; DIEP, deep inferior epigastric perforators; GEAV, gastroepiploic artery and vein; LD, latissimus dorsi; TRAM, transverse rectus abdominis myocutaneous.

\section{Supplementary Material}

Supplementary data.

http:/ / www.jcancer.org/v09p1782s1.pdf

\section{Acknowledgements}

This project was supported by the Science and Technology Department of Zhejiang Province (grant number 2015C33097), the Natural Science Foundation of China (grant number 81502463), and the Natural Science Foundation of Zhejiang Province (grant number Y15H160053, Q15H070004, LY13H160039).

\section{Ethics approval and consent to participate}

This work has received written consent from all the patients and has been approved by the ethics committee of Zhejiang Provincial People's Hospital.

\section{Authors' contributions}

Chao Ni, Zhengye Lv and Xiangyang Song conceived and designed the study. Ziguan Zhu, Qingping Xie, Chao Ni, Zhengye Lv and Xiangyang Song participated in the surgery. Yin Xin, Qingping Xie, Hongjun Yuan and Xiaoyan Zhu performed the literature identification and data collection. Chao $\mathrm{Ni}$, Miaochun Zhong and Wenjie Xia analysed the data and performed the statistical analysis. Zhengye Lv and Xiangyang Song critically revised the manuscript. All authors commented on drafts of the paper and approved the final manuscript.

\section{Competing Interests}

The authors have declared that no competing interest exists.

\section{References}

[1] Veronesi U, Cascinelli N, Mariani L, Greco M, Saccozzi R, Luini A, et al. Twenty-year follow-up of a randomized study comparing breast-conserving surgery with radical mastectomy for early breast cancer. The New England journal of medicine. 2002;347:1227-32.

[2] Riba J, de Romani SE and Masia J. Neoadjuvant Chemotherapy for Breast Cancer Treatment and the Evidence-Based Interaction with Immediate Autologous and Implant-Based Breast Reconstruction. Clinics in plastic surgery. 2018;45:25-31.

[3] van Alphen TC, Fechner MR, Smit JM, Slooter GD and Broekhuysen CL. The laparoscopically harvested omentum as a free flap for autologous breast reconstruction. Microsurgery 2017;37:539-45.

[4] Claro F, Jr., Sarian LO and Pinto-Neto AM. Omentum for Mammary Disorders: A 30-Year Systematic Review. Annals of surgical oncology. 2015;22:2540-50

[5] dos Santos Costa S, Blotta RM, Mariano MB, Meurer L and Edelweiss MI. Aesthetic improvements in Poland's syndrome treatment with omentum flap. Aesthetic plastic surgery. 2010;34:634-9.

[6] Guan D, Lin H, Lv Z, Xin Y, Meng K and Song X. The oncoplastic breast surgery with pedicled omental flap harvested by laparoscopy: initial experiences from China. World journal of surgical oncology. 2015;13:95.

[7] Kroll SS and Baldwin B. A comparison of outcomes using three different methods of breast reconstruction. Plastic and reconstructive surgery. 1992;90:455-62.

[8] Rose MA, Olivotto I, Cady B, Koufman C, Osteen R, Silver B, et al. Conservative surgery and radiation therapy for early breast cancer. Long-term cosmetic results. Archives of surgery. 1989;124:153-7.

[9] Kim H, Yoon CS, Lee HB, Ko BK, Kim GY and Kim KN. Mini-breast reconstruction with an omental flap: a retrospective clinical study. ANZ journal of surgery. 2016. 
[10] Jimenez AG, St Germain P, Sirois M, Hatheway M and Lethbridge R. Free omental flap for skin-sparing breast reconstruction harvested laparoscopically. Plastic and reconstructive surgery. 2002;110:545-51.

[11] Phillips CM. Breast reconstruction after classic radical mastectomy: use of omental pedicled grafts and fascia lata. Southern medical journal. 1982;75:270-3.

[12] McColl I. Reconstruction of the breast with omentum after subcutaneous mastectomy. Lancet. 1979;1:134-5.

[13] Jurkiewicz MJ and Arnold PG. The omentum: an account of its use in the reconstruction of the chest wall. Annals of surgery. 1977;185:548-54.

[14] Khater A. Evaluation of pedicled omental flap delivered through a minilaparotomy for immediate breast reconstruction in obese patients. Aesthetic plastic surgery. 2013;37:1140-5.

[15] Zaha H, Abe N, Sagawa N and Unesoko M. Oncoplastic surgery with omental flap reconstruction: a study of 200 cases. Breast cancer research and treatment. 2017;162:267-74.

[16] Li N, Zheng Z, Li J, Fan J, Wang $\mathrm{T}$, Zhang J, et al. Immediate breast reconstruction with omental flap for luminal breast cancer patients: Ten clinical case reports. Medicine. 2017;96(e):7797.

[17] Zhang P, Luo Y, Deng J, Shao G, Han S and Huang Z. Endoscopic axillary lymphadenectomy combined with laparoscopically harvested pedicled omentum for immediate breast reconstruction. Surgical endoscopy. 2015;29:1376-83.

[18] Cothier-Savey I, Tamtawi B, Dohnt F, Raulo Y and Baruch J. Immediate breast reconstruction using a laparoscopically harvested omental flap. Plastic and reconstructive surgery. 2001;107:1156-63.

[19] HAN Ming-yue YW-J, QIU Xiao-dong, WANG Hong-wu, GUO Jun-jie, LIU Cui-ping. Pedicle greater omentum dissection through a small transverse abdominal incision in an immediate breast reconstruction. Chin J Breast Dis. 2012;6:649-55.

[20] $\mathrm{Yu}$ X. First stage breast reconstruction with laparoscopic harvested pedicled-omental flap after modified radical mastectomy with nipple-areola preserved: 54 cases reports. Chinese Journal of Woman and Child Health Research. 2016;27:331.

[21] Macadam SA, Bovill ES, Buchel EW and Lennox PA. Evidence-Based Medicine: Autologous Breast Reconstruction. Plastic and reconstructive surgery. 2017;139:204e-29e

[22] Zaha H, Sunagawa H, Kawakami K, Touyama T, Yonaha T and Ohshiro N. Partial breast reconstruction for an inferomedial breast carcinoma using an omental flap. World journal of surgery. 2010;34:1782-7.

[23] Romanini MV, Vidal C, Godoy J and Morovic CG. Laparoscopically harvested omental flap for breast reconstruction in Poland syndrome. Journal of plastic, reconstructive \& aesthetic surgery: JPRAS. 2013;66:e303-9.

[24] Zaha H. Oncoplastic volume replacement technique for the upper inner quadrant using the omental flap. Gland surgery. 2015;4:263-9.

[25] Zaha H and Inamine S. Laparoscopically harvested omental flap: results for 96 patients. Surgical endoscopy. 2010;24:103-7.

[26] Carter JC and Church FC. Mature breast adipocytes promote breast cancer cell motility. Experimental and molecular pathology. 2012;92:312-7.

[27] Lohsiriwat V, Curigliano G, Rietjens M, Goldhirsch A and Petit JY. Autologous fat transplantation in patients with breast cancer: "silencing" or "fueling" cancer recurrence? Breast. 2011;20:351-7. 